\title{
Assessment of the incidence of temporomandibular disorders in patients diagnosed with psoriasis
}

\author{
Małgorzata Stodółkiewicz ${ }^{1, A-B, D, F \oplus}{ }^{\infty}$, Monika Turska ${ }^{2,3, A, C-F \oplus}$, Jacek Szkutnik ${ }^{1, A-B, D-F} \oplus$, \\ Leszek Szalewski ${ }^{4, A, C-F} \odot$ \\ 1 Department of Functional Masticatory Disorders, Medical University, Lublin, Poland \\ ${ }^{2}$ Doctoral Student at Department of Pharmacology, Faculty of Nursing and Health Sciences, Medical University, Lublin, \\ Poland \\ ${ }^{3}$ Med-Laser Non-Public Health Care Centre, Lublin, Poland \\ ${ }^{4}$ Department of Dental Prosthetics, Medical University, Lublin, Poland \\ A - Research concept and design, B - Collection and/or assembly of data, C - Data analysis and interpretation, \\ $D$ - Writing the article, E-Critical revision of the article, F- Final approval of article
}

Stodółkiewicz M, Turska M, Szkutnik J, Szalewski L. Assessment of the Incidence of Temporomandibular Disorders in Patients Diagnosed with Psoriasis. J Pre Clin Clin Res. 2019; 13(3): 110-113. doi: 10.26444/jpccr/112336

\begin{abstract}
Introduction and objective. Temporomandibular disorders (TMD) are currently among the most commonly diagnosed disorders of the stomatognathic system. They are related to the overall condition of temporomandibular joints (TMJ). In recent years, there have been an increasing number of reports related to clinical patients suffering from stomatognathic disorders accompanying psoriasis. The aim of the study is to assess the incidence and identify the types of temporomandibular disorders in patients diagnosed with psoriasis.

Materials and method. The study was conducted in a group of 64 patients ( 32 men and 32 women) treated for psoriasis at the Med-Laser Non-Public Health Care Centre in Lublin. The study was conducted in two stages. The first stage entailed the use of a normalised questionnaire in which the patients were surveyed regarding the incidence of TMD symptoms. The survey questions were prepared on the basis of the Research Diagnostic Criteria for Temporomandibular Disorders (RDC/ TMD). The second stage entailed the performance of clinical examinations in accordance with the RDC/TMD classification guidelines.

Results. The survey revealed that over $1 / 3$ of the subjects experienced at least one type of TMJ disfunction included in the RDC/TMC questionnaire.

Conclusions. Psoriasis patients are most often classified into class Ila TMD according to RDC / TMD diagnoses. For this reason, patients reporting such symptoms should be referred for full diagnostic stomatological examination for the possible presence of temporomandibular disorders. The inclusion of screening for TMD symptoms should be considered in patients treated for psoriasis.
\end{abstract}

\section{Key words}

psoriasis, psoriatic arthritis, temporomandibular joint, temporomandibular joint disorders, TMJ pain

\section{INTRODUCTION}

Temporomandibular disorders (TMD) are currently among the most commonly diagnosed disorders of the stomatognathic system. Pain originating from the joints of the stomatognathic system is the third, after dental caries and periodontal disease, the most common problem mentioned by patients reporting for stomatological treatment. Patients also very often report acoustic symptoms in the temporomandibular joints, mainly clicking or crackling sounds [1]. According to the relevant epidemiological studies, TMD symptoms are observed in approx. 60-80\% of the population. As follows from the epidemiological data, pain associated with TMD affects approx. $9-13 \%$ of the population, including almost $50 \%$ more female than male patients. Symptoms are typically exacerbated in patients aged between 20-40 years and include: incorrect mandibular

Address for correspondence: Leszek Szalewski, Department of Dental Prosthetics, Medical University, Lublin, Poland

E-mail: Leszek.Szalewski@umlub.pl

Received: 29.05.2019; accepted: 13.09.2019; first published: 20.09.2019 mobility (reduced or excessive range of motion), mandibular deviation (while opening or closing the jaw) acoustic symptoms in the temporomandibular joint (crackling, clicking), mandibular head skipping, and pain in the cephalic and facial region (joint and muscle pain). Accompanying symptoms can also include: pathological dentalgia, noncarious defects, gomphiasis (dental displacement), masseter muscle hypertrophy, morning stiffness of facial muscles, dental numbness and/or tingling, as well as symptoms in the visual and auditory systems, e.g. ear or eye pain, a sense of obstruction in the ears, tinnitus, vertigo, a sense of the eyeball bulging out of its socket, and lacrimation for no apparent reason. Disorders of the temporomandibular joint can also cause symptoms to occur in more distant regions of the body, e.g. spinal pain, lower back pain (sacralgia, lumbago), coxalgia, as well as knee or even ankle pain. $92 \%$ of all headaches treated as migraines originate from existing TMD.

Temporomandibular disorders have a complex aetiology. Both local and general factors seem to play a significant role in the same. The most commonly identified local causes 
include incorrect occlusion and parafunctional activity (e.g. clenching, clamping teeth together, compulsive biting down or chewing), while the most common general causes include stress and abnormal deep afferent impulsation.

Psoriasis is an inflammatory, non-contagious disease of a chronic and recurrent character. According to various sources, the incidence of psoriasis in the global adult population is estimated at approx. 1-3\% [3] while the incidence in children is considerably less. Epidemiological data tend to vary significantly depending on the country as well as relative to geographical and racial factors. Psoriasis can occur at any age, although two peak periods can be identified in this respect - it most commonly occurs in young adults between 20-30 years of age, as well as in patients aged approx. 50-60.

The exact causes of psoriasis have yet to be clearly identified. Some research data suggest a genetic origin with a particular focus of the human leukocyte antigen (HLA) system. However, heredity is a multifaceted phenomenon and the emergence of the disease is conditional upon numerous complex processes that need to occur simultaneously. Research is also now being conducted with the aim of identifying immunological factors which may have an influence on the development and incidence of psoriasis, including the contribution of $\mathrm{T}$ lymphocytes and the mediators they produce. Various external and internal stimuli may also cause the onset of symptoms. These include physical (surgeries, tattooing, vaccinations, excoriations) as well chemical factors (chemical burns, cosmetics). Internal factors include: streptococcal pharyngitis, which often precedes the original dissemination in children and young adults, stress or HIV infection. It should be emphasised that exacerbation of the symptoms is commonly observed after chronic pharmacotherapy. This is most commonly observed in the case of broadly administered arterial hypertension drugs, e.g. beta adrenolytic [4], as well as non-steroidal ant-inflammatory drugs (NSAIDs), often taken by patients without medical consultation.

The course of psoriasis can vary significantly between respective patients. The scalp is one of the most common locations [5]. Another area often affected by the disease is the intertriginous region (intragluteal cleft, groin, axilla, navel), which is due to the heat, moist, and continued irritation of the skin in those areas. The severity of skin lesions can be limited but they can also proliferate to significant areas of the skin, in many cases up to $90 \%$ of the skin area in conditions referred to as erythroderma. The first lesions are usually erythrodermic or plaque in character, often with accompanying silvery scaling. The lesions can cause itching, burning, or even pain. Traditionally, psoriasis was considered as a disease manifesting itself only externally; however, recent studies have revealed an increased incidence of serious cardiovascular disorders in patients diagnosed with psorias, compared to the general population [6]. A similar correlation was observed regarding the increased incidence of chronic kidney disease [7]. Furthermore, chronic psoriasis constitutes a serious psychological problem due to the difficult treatment, common relapses, as well as lack of social acceptance for skin lesions visible on the patient's skin, all of which increase the risk of depression [8].

Psoriasis is usually categorised as one of two main types of psoriasis or psoriatic arthritis. Type 1 psoriasis usually develops in patients under the age of 40 . It is believed to be strongly correlated with the HLA system, often occuring in members of the same family, and its course tends to be exacerbated to severe levels as the disease progresses.

Type 2 psoriasis is usually diagnosed in patients over the age of 40 . It is believed that it is not hereditary, has no strong correlations with the HLA system, and more commonly affects the areas of the nails and joints.

\section{OBJECTIVE}

The aim of the study was to assess the incidence and identify the types of TMD observed in patients diagnosed with psoriasis.

\section{MATERIALS AND METHOD}

The study was conducted in a group of 64 patients ( 32 men and 32 women) treated for psoriasis at the Med-Laser Non-Public Health Care Centre in Lublin between March - September 2018. The study group patients were aged between 13-66, with the mean age of 44 years and median of 46.13 patients (20.31\%) were between 13 -29-years-old, 8 patients $(12.3 \%)$ $30-39,12$ patients (18.75\%) 40-49, 15 (23.44\%) were 50-59, and $16(25 \%)$ were $60-66$-years-old. $59.38 \%$ of the patients were treated for type 1 cutaneous psoriasis, $10.94 \%$ for type 2 cutaneous psoriasis, and the remaining $29.69 \%$ of patients suffered from psoriatic arthritis. Inclusion criteria were: over 12 years of age (to avoid mixed dentition), psoriasis was the main disease and good mental state of the patient. Exclusion criteria were poor general condition of the patient and no desire to participate in the study.

The study consisted of two stages. The first stage entailed the use of a normalised questionnaire in which the patients were surveyed regarding the incidence of TMD symptoms. The survey questions were prepared on the basis of the Research Diagnostic Criteria for Temporomandibular Disorders (RDC/TMD) [2]. The questions referred to: joint pain, muscle pain, incidence of acoustic symptoms, and functional disorders of the mandible. Patients were also surveyed in terms of the incidence of pain in the region of temporomandibular joints during the preceding month, reduced range of jaw mobility, clicking, crackling, teeth clenching at night, teeth clenching during the day, dental stiffness and/or pain in the morning, ringing or buzzing in the ears, occlusal discomfort, swelling, pain in joints other than TMJ, as well as incidence of headaches in the preceding 6 months.

The second stage of the study involved clinical examinations in accordance with the RDC/TMD classification guidelines. The intraoral clinical examination entailed a general dental examination and assessment of any prosthetics in terms of restoration of correct dental function. The functional clinical examination entailed: extraoral palpation of muscles in the stomatognathic, neck and shoulder girdle region, intraoral palpation of stomatognathic muscles, palpation of the lateral and distal regions of temporomandibular joint (TMJ), examination for audible symptoms and muscle pain during slow movements of the mandible, and a clinical analysis of mandibular mobility: range and sequence of abduction motion including any deviations, range and sequence of lateral motion, range and sequence of protrusion and retrusion, as well as potential dislocation of the TMJ 
articular disc on the basis of the observed acoustic symptoms, and diagnostic tests. All procedures performed the studies involving the participants were in accordance with the ethical standards of the Institutional and/or National Research Committee and with the 1964 Helsinki Declaration and its later amendments, or comparable ethical standards. The authors obtained the informed consent of patients/caregivers of patients to participate in the study.

The obtained results were processed and classified in accordance with the RDC/TMD classification guidelines. Statistical analysis of the results was conducted with the use of Fisher's test. The value of $\mathrm{p}<0.05$ was adopted as the threshold of statistical significance.

\section{RESULTS}

The survey revealed that $37.5 \%$ of the analysed patients experienced at least one of the TMD symptoms included in the questionnaire. Pain in the TMJ region was reported by $17.19 \%$ of the respondents, 4 men and 7 women. Muscle pain and acoustic symptoms were reported by $4.69 \%$ of the patients, while mandibular mobility disorders were present in $3.13 \%$ of female respondents. $41.67 \%$ of patients experiencing TMD admitted that they needed treatment in this respect. $45.31 \%$ of the respondents reported having experienced headaches in the period of 6 months prior to the survey.

On the basis of the clinical study conducted in accordance with the RDC/TMD classification (Tab. 1), TMD was diagnosed in $9.37 \%$ of the patients, with class 2 a diagnosed in $7.81 \%$ and $2 b$ in $1.56 \%$ of the cases, while in the remaining $90.63 \%$ of the patients, TMD was not diagnosed. Figures 1 and 2 present the occurrence of TMD symptoms and selected

Table 1. Classification of temporomandibular disorders in accordance with RDC/TMD

\begin{tabular}{l}
\hline I-Muscular form \\
a- Myofascial pain \\
b- Myofascial pain with reduced jaw mobility \\
\hline II- Form with articular disc disorders \\
\hline a- Disc displacement with reduction \\
b- Disc displacement without reduction and with reduced jaw mobility \\
c- Disc displacement without reduction and without reduced jaw mobility \\
III-Form with arthritis \\
a- Joint pain \\
b- Temporomandibular arthritis \\
c- Temporomandibular arthrosis
\end{tabular}

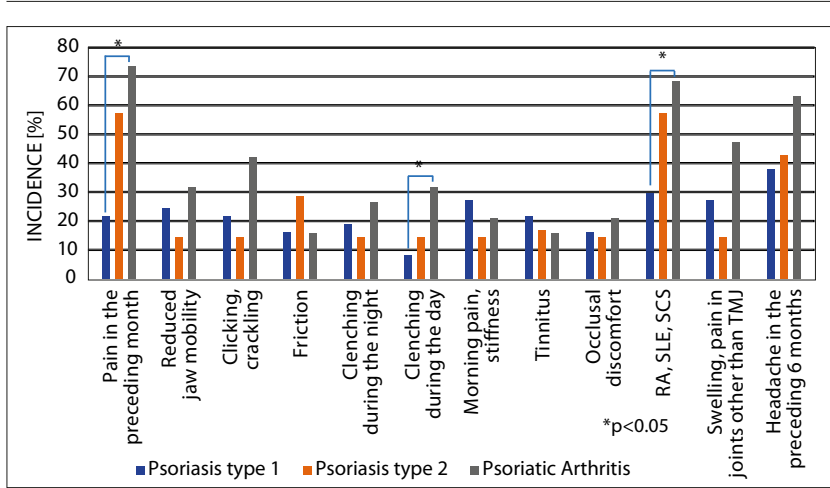

Figure 1. Incidence of Temporomandibular Disorders symptoms and selected risk factors in patients relative to the type of psoriasis. (RA - Rheumatoid Arthritis; SLE - systemic lupus erythematosus; SCS - systemic joint diseases)

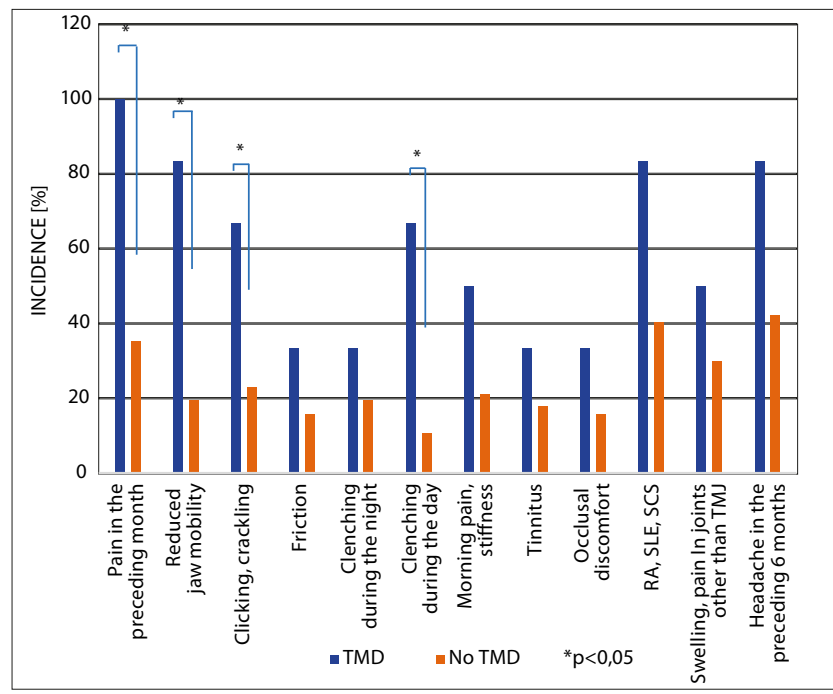

Figure 2. Incidence of TMD symptoms and selected risk factors in patients diagnosed and undiagnosed with TMD. (RA - Rheumatoid Arthritis; SLE - systemic lupus erythematosus; SCS - systemic joint diseases)

risk factors in patients relative to the type of psoriasis and TMD diagnosis.

Almost half (43.75\%) of the patients had complete alveolar arches or correct prosthetic restorations performed, the missing teeth in the remaining patients were either unrestored or the prosthetic restorations were found to be incorrect.

\section{DISCUSSION}

It has been estimated that between 1.3 to even $34.7 \%$ of patients diagnosed with psoriasis develop chronic arthritis (psoriatic arthritis), which can lead to significant articular deformations, and in extreme cases to disability. The disease usually takes the form of asymmetric inflammation of individual joints, typically distal digital joints. The affected digit becomes dactylitic, a state described as 'sausage fingers', while x-ray examinations produce the characteristic 'pencilin-cup' images. The disease can also extend to the knees, ankles, and spinal joints. In a study by Dervis and Dervis, it was reported that temporomandibular disorders did not occur more frequently in healthy patients compared to patients diagnosed with psoriasis. At the same time, they observed a high incidence of disorders in patients diagnosed with psoriatic arthritis (PA) [9].

Numerous clinical cases have been reported in recent years where psoriatic patients also suffered from temporomandibular disorders. Okkesim et al. described the case of a patient diagnosed with psoriasis who struggled with pain and reduced mobility of the TMJ [10]. The performed $\mathrm{X}$-ray (pantograph) with CBCT revealed foci of erosion and abnormal (rough) edges of the articular head surfaces. Similar erosion and osteoporotic lesions were revealed on the surface of the temporal bone. On this basis, psoriatic arthritis was diagnosed and standard TMD treatment was recommended, i.e. administration of NSAIDs and splint therapy. The symptoms subsided after one month of therapy. The authors emphasise a $5-8 \%$ risk of PA in patients diagnosed with psoriasis, which in turn can lead to TMD.

Loster reports the incidence of TMD in a group of young Polish adults at approx. 26.5\% [11]. In the age group of 
students, the incidence of TMD was within the range from $15-68.6 \%$ [12-17], and in the group of adults it wa estimated at between $15-50.5 \%$ [18-19]. The incidence of TMD in psoriasis patients is approx. $10 \%$, which is significantly below the levels reported in literature for the general population. The discrepancy remains unexplained although it may be due to the fact that the incidence of TMD in the elderly population decreases is as low as 3-6\% [20-22], and that the mean age of the study group of patients was 44 .

The symptoms most commonly reported by psoriasis patients diagnosed with TMD in accordance with RDC classification included: pain in the preceding month, reduced jaw mobility and headaches (over $80 \%$ ) as well as clicking, crackling, clenching during the day (over 60\%). Psoriasis patients without TMD most commonly reported headaches in the preceding 6 months, pain in the preceding month, as well as swelling, pain in joints other than TMJ (20-40\%). Psoriasis patients diagnosed with TMD suffered - significantly more frequently than psoriasis patients without TMD - from: pain in the preceding month, reduced jaw mobility, clicking and crackling, as well as clenching during the day (Fig. 2).

In own study, psoriasis patients were divided into 3 subgroups composed of patients suffering from: type 1 psoriasis, type 2 psoriasis, and psoriatic arthritis. Symptoms of TMJ disorders were most often observed in patients suffering from PA. Other commonly reported symptoms included pain in the preceding month and headache in the preceding 6 months (over 60\%). A juxtaposition of the incidence of TMJ symptoms in the aforementioned three groups of psoriasis patients revealed a statistically significantly higher incidence of pain in the preceding month and clenching during the day in patients suffering from psoriatic arthritis, compared to type 1 psoriasis patients. In the group of patients with type 2 psoriasis, no statistically significant differences were observed, most likely due to the low number of patients in this group (Fig. 1). It seems that the described symptoms may be helpful in referring patients for dental examinations with a particular indication of possible TMD.

\section{CONCLUSIONS}

The incidence of TMD in examined psoriasis patients is approx. $10 \%$ and is lower than that reported in the general population. This can be attributed to the effects of psoriasis treatment received by the patients. Psoriasis patients are most often classified into class IIa TMD according to RDC/TMD diagnoses. For this reason, patients reporting such symptoms should be referred for full diagnostic stomatological examination for the possible presence of temporomandibular disorders.

The inclusion of screening for TMD symptoms should be considered in patients treated for psoriasis.

\section{REFERENCES}

1.Berger M, Szkutnik J, Szalewski L, Wójcik D, Bakalczuk M, Ginszt M. Correlation between generalized joint laxity and symptoms of temporomandibular disorders. Pol Med. J. 2016; XL(238): 248-251.
2. Osiewicz MA, Lobbezoo F, Loster BW, Wilkosz M, Naeije M, Ohrbach R. Research Diagnostic Criteria for Temporomandibular Disorders (RDC/ TMD) - The Polish version of a dual-axis system for the diagnosis of TMD. RDC/TMD Form. J Stoma 2013; 66, 5: 576-649.

3.Zhu JF, Kaminski MJ, Pulitzer DR, Hu J, Thomas HF. Psoriasis: pathophysiology and oral manifestations. Oral diseases. 1996; 2(2): $135-44$.

4. Wu S, Han J, Li WQ, Qureshi AA. Hypertension, antihypertensive medication use, and risk of psoriasis. JAMA dermatology. 2014; 150(9): 957-63.

5. Burgdorf W, Wolff H, Landthaler M. Redakcja wydania polskiego: Gliński W, Czarnecka-Operacz M, Krasowska D, Serwin A, Wolska H. Dermatologia Braun-Falco. 3 ed. Lublin 2017.

6. Gelfand JM, Neimann AL, Shin DB, Wang X, Margolis DJ, Troxel AB. Risk of myocardial infarction in patients with psoriasis. Jama. 2006; 296(14): 1735-41.

7. Wan J, Wang S, Haynes K, Denburg MR, Shin DB, Gelfand JM. Risk of moderate to advanced kidney disease in patients with psoriasis: population based cohort study. BMJ (Clinical research ed). 2013; 347: f5961.

8. Kurd SK, Troxel AB, Crits-Christoph P, Gelfand JM. The risk of depression, anxiety, and suicidality in patients with psoriasis: a population-based cohort study. Archives of dermatology. 2010; 146(8): 891-5.

9. Dervis E, Dervis E. The prevalence of temporomandibular disorders in patientswith psoriasis with or without psoriatic arthritis. Journal of Oral Rehabilitation 2005; 32: 786-793.

10. Okkesim A, Adisen MZ, Misirlioglu M. Temporomandibular joint involvement in psoriatic arthritis. Niger J Clin Pract 2017; 20: 1501-4.

11.Loster JE, Osiewicz MA, Groch M, Ryniewicz W, Wieczorek A. The prevalence of TMD in Polish young adults. J Prosthodont. 2017; 26: 284-288.10.1111/jopr.2017.26.issue-4

12. Bonjardim L, Lopes-Filho R, Amado G, Albuquerque R, Gonçalves S. Association between symptoms of temporomandibular disorders and gender, morphological occlusion and psychological factors in a group of university students. Indian J Dent Res, 20 (2009), pp. 190-194.

13. Conti P, Ferreira P, Pegoraro L, Conti J, Salvador M. A crosssectional study of prevalence and etiology of signs and symptoms of temporomandibular disorders in high school and university students. J Orofac Pain. 1996; 10: 254-62.

14. Ebrahimi M, Dashti H, Mehrabkhani M, Arghavani M, DaneshvarMozafari A. Temporomandibular disorders and related factors in a group of Iranian adolescents: a cross-sectional survey. J Dent Res Dent Clin Dent Prospect. 2011; 5: 123-7.

15. Oliveira A, Bevilaqua-Grossi D, Dias E. Signs and symptoms of temporomandibular disorders across Brazilian regions. Fisioter. Pesqui. 2008; 15(4): 392-6.

16. Pedroni C, De Oliveira A, Guaratini M. Prevalence study of signs and symptoms of temporomandibular disorders in university students. J Oral Rehabil. 2003; 30: 283-9.

17. Rani S, Pawah S, Gola S, Bakshi M. Analysis of Helkimo index for temporomandibular disorder diagnosis in the dental students of Faridabad city: a cross-sectional study. J Indian Prosthodont Soc. 2017; 17: 48-52.

18. Sampaio N, Oliveira M, Ortega A,Santos L, Alves T. Temporomandibular disorders in elderly individuals: the influence of institutionalization and sociodemographic factors. Codas. 2017; 29(2): e20160114, 2017.

19. Yadav S, Yang Y, Dutra EH, Robinson JL, Wadhwa S. Temporomandibular Joint Disorders in Older Adults. J Am Geriatr Soc. 2018; 5: 2. doi: 10.1111/ jgs. 15354

20. Horst OV, Cunha-Cruz J, Zhou L, Manning W, Mancl L, DeRouen TA. Prevalence of pain in the orofacial regions in patients visiting generaldentists in the Northwest Practice-based Research Collaborative in Evidence-based Dentistry research network. J Am Dent Assoc. 2015; 146: 721-8.

21. Maixner W, Fillingim RB, Williams DA, Smith SB, Slade GD. Overlappingchronic pain conditions: Implications for diagnosis and classification. J Pain 2016; 17: T93-T107.

22. Slade GD, Bair E, By K, Mulkey F, Baraian C, Rothwell R, et al. Study methods, recruitment, sociodemo-graphic findings, and demographic representativeness in the OPPERA study. J Pain 2011; 12: T12-T26. 